Irish Math. Soc. Bulletin

Number 78, Winter 2016, 25-30

ISSN 0791-5578

\title{
A Mean Value Inequality for Euler's Beta Function
}

\author{
HORST ALZER AND RICHARD B. PARIS
}

Abstract. Let $B(x, y)$ be Euler's beta function. We prove that the inequalities

$$
0<\frac{B\left(\sqrt{x y}, \frac{x+y}{2}\right)}{B(x, y)}<1
$$

hold for all $x, y>0$ with $x \neq y$. The given constant bounds are best possible. This result is extended to the case when the beta function in the numerator has arguments given by the weighted geometric and arithmetic means.

\section{INTRODUCTION}

The beta function, also known as the Eulerian integral of the first kind, is defined for positive real numbers $x$ and $y$ by

$$
B(x, y)=\int_{0}^{1} t^{x-1}(1-t)^{y-1} d t=\frac{\Gamma(x) \Gamma(y)}{\Gamma(x+y)},
$$

where $\Gamma$ denotes the classical gamma function. From the product representation of $1 / \Gamma(x)$ [6, Eq. (1.1.9)], it follows that

$$
B(x, y)=\frac{x+y}{x y} \prod_{n=1}^{\infty}\left(1+\frac{x y}{n(x+y+n)}\right)^{-1} .
$$

The beta function plays an important role in the theory of special functions and it also has remarkable applications in physics, stochastic processes and other fields. A collection of the main properties of $B(x, y)$ as well as interesting historical comments on this subject can be found, for instance, in [6].

In the recent past, several research papers have appeared providing various inequalities for the beta function and its relatives. We refer to $[1-5],[7,9,10,11]$ and the references cited therein. For example,

2010 Mathematics Subject Classification. 33B15, 26D07, 26E60.

Key words and phrases. Beta function, mean values, inequalities.

Received on 30-5-2016; revised 31-7-2016. 
for all $x, y>0$ we have that $B(x, y)$ separates the geometric and arithmetic means of $B(x, x)$ and $B(y, y)$, that is,

$$
\sqrt{B(x, x) B(y, y)} \leq B(x, y) \leq \frac{B(x, x)+B(y, y)}{2} .
$$

The first inequality is given in [9], whereas a proof for the second one can be found in [3]. In this note we present a new mean value inequality for the ratio of two beta functions.

Theorem. For all positive real numbers $x$ and $y$ with $x \neq y$ we have

$$
0<\frac{B\left(\sqrt{x y}, \frac{x+y}{2}\right)}{B(x, y)}<1
$$

Both constant bounds are sharp.

\section{A Lemma}

In order to prove the right-hand side of (3) we apply a lemma which offers an upper bound for the ratio given in (3) in terms of geometric and arithmetic means.

Let $x, y>0$ and $w \in(0,1)$. The weighted geometric and arithmetic means are defined by

$$
G_{w}(x, y)=x^{w} y^{1-w} \quad \text { and } \quad A_{w}(x, y)=w x+(1-w) y .
$$

Moreover, we set

$$
G=G_{1 / 2}(x, y)=\sqrt{x y} \quad \text { and } \quad A=A_{1 / 2}(x, y)=\frac{x+y}{2} .
$$

Lemma. Let $v, w \in(0,1)$. The inequality

$$
\frac{B\left(G_{v}(x, y), A_{w}(x, y)\right)}{B(x, y)} \leq \frac{1}{2}\left[\left(\frac{G_{v}(x, y)}{A_{w}(x, y)}\right)^{2}+\frac{G_{v}(x, y)}{A_{w}(x, y)}\right]
$$

holds for all $x, y>0$ if and only if $v=w=1 / 2$.

Proof. First, we assume that (4) is valid for all $x, y>0$. Let

$$
F_{v, w}(x, y)=2 \frac{B\left(G_{v}(x, y), A_{w}(x, y)\right)}{B(x, y)}\left[\left(\frac{G_{v}(x, y)}{A_{w}(x, y)}\right)^{2}+\frac{G_{v}(x, y)}{A_{w}(x, y)}\right]^{-1} .
$$

Then, we have for $x, y>0$ :

$$
F_{v, w}(x, y) \leq 1=F_{v, w}(y, y) .
$$

Use of

$$
\frac{\partial}{\partial x} B(x, y)=B(x, y)[\psi(x)-\psi(x+y)]
$$


where $\psi=\Gamma^{\prime} / \Gamma$ is the logarithmic derivative of the gamma function, yields

$$
0=\left.2 y \frac{\partial}{\partial x} F_{v, w}(x, y)\right|_{x=y}=3(w-v)+2(v+w-1) y(\psi(y)-\psi(2 y)) .
$$

We denote the expression on the right-hand side of $(5)$ by $H_{v, w}(y)$. Since

$$
\lim _{t \rightarrow 0+} t \psi(t)=-1 \quad \text { and } \quad \psi(1)-\psi(2)=-1,
$$

we obtain

$$
\lim _{y \rightarrow 0+} H_{v, w}(y)=1-4 v+2 w=0
$$

and

$$
H_{v, w}(1)=2-5 v+w=0 .
$$

This leads to $v=w=1 / 2$.

Next, we prove (4) with $v=w=1 / 2$. Application of (2) leads to

$$
B(x, y)=\frac{2 A}{G^{2}} \prod_{n=1}^{\infty}\left(1+\frac{G^{2}}{n(2 A+n)}\right)^{-1}
$$

and

$$
\frac{B(G, A)}{B(x, y)}=\frac{G(G+A)}{2 A^{2}} \prod_{n=1}^{\infty} f_{n}
$$

with

$$
f_{n}=\left(1+\frac{G^{2}}{n(2 A+n)}\right)\left(1+\frac{G A}{n(G+A+n)}\right)^{-1} .
$$

Since $A-G \geq 0$, we obtain

$$
f_{n}=1-\frac{G(A-G)(G+2 A+n)}{(G+n)(A+n)(2 A+n)} \leq 1 \quad \text { for } \quad n \geq 1 .
$$

Therefore,

$$
\frac{B(G, A)}{B(x, y)} \leq \frac{G(G+A)}{2 A^{2}}=\frac{1}{2}\left[\left(\frac{G}{A}\right)^{2}+\frac{G}{A}\right] .
$$

This establishes (4) with $v=w=1 / 2$.

Remark 2.1. If $x \neq y$, then $A-G>0$, so that (8) gives $f_{n}<1$ for $n \geq 1$. This implies that (9) holds with " $<$ " instead of " $\leq$ ". Thus, if $v=w=1 / 2$, then the sign of equality is valid in (4) if and only if $x=y$.

We are now in a position to establish our main result. 


\section{Proof of Theorem}

An application of (4) with $v=w=1 / 2$ yields for $x, y>0$ with $x \neq y$ :

$$
\frac{B(G, A)}{B(x, y)}<1-\frac{(A-G)(A+G / 2)}{A^{2}}<1 .
$$

It remains to show that the bounds 0 and 1 are best possible. We denote the ratio in $(3)$ by $R(x, y)$. Then,

$$
R(x, x)=1 .
$$

Use of the recurrence formula $\Gamma(x+1)=x \Gamma(x)$ and (1) gives

$$
R(x, 1)=\sqrt{x} \frac{\Gamma(\sqrt{x}+1) \Gamma((x+1) / 2)}{\Gamma(\sqrt{x}+(x+1) / 2)} .
$$

It follows that

$$
\lim _{x \rightarrow 0^{+}} R(x, 1)=0 .
$$

From (10) and (11) we conclude that the constant upper and lower bounds given in (3) cannot be improved.

Remark 3.1. Inequality (4) with $v=w=1 / 2$ reveals that

$$
\frac{A}{G} \leq \frac{B(x, y)}{B(G, A)}
$$

is valid for all $x, y>0$. This is a converse of the well-known arithmetic mean - geometric mean inequality $A / G \geq 1$. Many additional inequalities for arithmetic and geometric means as well as for numerous other mean values are given in the monograph [8].

It is natural to ask whether the right-hand side of (3) is valid for geometric and arithmetic means with a weight different from $1 / 2$. The following remark reveals that if both means have the same weight, then the answer is "no".

Remark 3.2. Let $w \in(0,1)$. The inequality

$$
B\left(G_{w}(x, y), A_{w}(x, y)\right) \leq B(x, y)
$$

holds for all $x, y>0$ if and only if $w=1 / 2$. We define

$$
I_{w}(x)=B(x, 1)-B\left(G_{w}(x, 1), A_{w}(x, 1)\right) .
$$

If (12) is valid for all $x, y>0$, then we obtain

$$
I_{w}(x) \geq 0=I_{w}(1) \quad \text { and } \quad I_{w}^{\prime}(1)=2 w-1=0 .
$$

Thus, $w=1 / 2$. 
Remark 3.3. If $w \in(0,1)$, then (12) holds for $x, y>0$ satisfying $G^{2} \leq G_{w}(x, y) A_{w}(x, y)$ or, equivalently, $G_{w}(y, x) \leq A_{w}(x, y)$. To see this we observe that an extension of (6) and (7) (we omit to display the $x, y$ dependence of $G_{w}$ and $\left.A_{w}\right)$ shows that

$$
\frac{B\left(G_{w}, A_{w}\right)}{B(x, y)}=Q_{w} \prod_{n=1}^{\infty} g_{n}(w), \quad Q_{w}=\frac{G^{2}\left(G_{w}+A_{w}\right)}{2 A G_{w} A_{w}}
$$

where

$$
\begin{gathered}
g_{n}(w)=\left(1+\frac{G^{2}}{n(2 A+n)}\right)\left(1+\frac{G_{w} A_{w}}{n\left(G_{w}+A_{w}+n\right)}\right)^{-1} \\
=1-\frac{n\left(G_{w} A_{w}-G^{2}\right)+2 A G_{w} A_{w}\left(1-Q_{w}\right)}{(2 A+n)\left(G_{w}+n\right)\left(A_{w}+n\right)} .
\end{gathered}
$$

Since

$$
\begin{gathered}
\frac{G^{2}\left(G_{w}+A_{w}\right)}{G_{w}}=G^{2}+G_{1-w} A_{w} \leq A_{w}\left(G_{w}+G_{1-w}\right) \\
\leq A_{w}\left(A_{w}+A_{1-w}\right)=2 A A_{w},
\end{gathered}
$$

where we have employed the arithmetic mean - geometric mean inequality $G_{w} \leq A_{w}$, we see that $Q_{w} \leq 1$ and $g_{n}(w) \leq 1$ for $n \geq 1$, whence the result follows.

Acknowledgement. We thank the referee for some helpful comments and for pointing out the extension given in Remark 3.3 .

\section{REFERENCES}

[1] P. Åhag, R. Czyż: An inequality for the beta function with application to pluripotential theory, J. Inequal. Appl. 2009, Article ID 901397, 8 p.

[2] H. Alzer: Sharp inequalities for the beta function, Indag. Math. 12 (2001), $15-21$.

[3] H. Alzer: Some beta-function inequalities, Proc. Roy. Soc. Edinb. 133A (2003), 731-745.

[4] H. Alzer: Inequalities for the beta function of n variables, ANZIAM 44 (2003), 609-623.

[5] H. Alzer: Inequalities for the beta function, Anal. Math. 40 (2014), 1-11.

[6] G.E. Andrews, R. Askey, R. Roy: Special Functions, Cambridge Univ. Press, Cambridge, 1999.

[7] B.A. Bhayo, J. Sándor: On the inequalities for beta function, Notes Numb. Th. Disc. Math. 21 (2015), 1-7.

[8] P.S. Bullen, D.S. Mitrinović, P.M. Vasić: Means and Their Inequalities, Reidel, Dordrecht, 1988. 
[9] S.S. Dragomir, R.P. Agarwal, N.S. Barnett: Inequalities for beta and gamma functions via some classical and new integral inequalities, J. Inequal. Appl. 5 (2000), 103-165.

[10] P. Ivády: On a beta function inequality, J. Math. Ineq. 6 (2012), 333-341.

[11] P. Ivády: On a beta function inequality II, J. Math. Sci. Adv. Appl. 22 (2013), 27-37.

Horst Alzer received his Ph.D. from the University of Bonn and completed his Habilitation at the University of Würzburg. He is mainly interested in special functions and inequalities.

Richard B. Paris is an Emeritus Reader in mathematics at the University of Abertay Dundee. He previously worked for Euratom at the French Atomic Energy Commission in the thermonuclear fusion department. His main area of interest is in asymptotic analysis. He is the author of two chapters in the NIST Handbook of Mathematical Functions.

(Alzer) Morsbacher Str. 10, 51545 Waldbröl, Germany

(Paris) Division of Computing and Mathematics, University of AberTAY Dundee, DundeE DD1 1HG, UK

E-mail address, H. Alzer: h.alzer@gmx.de

E-mail address, R.B. Paris: R.Paris@abertay.ac.uk 